\title{
DESARROLLO DE LOS CIBERMEDIOS EN COLOMBIA
}

\author{
Elías Said-Hung, Carlos Arcila-Calderón y Jorge Méndez-Barraza
}

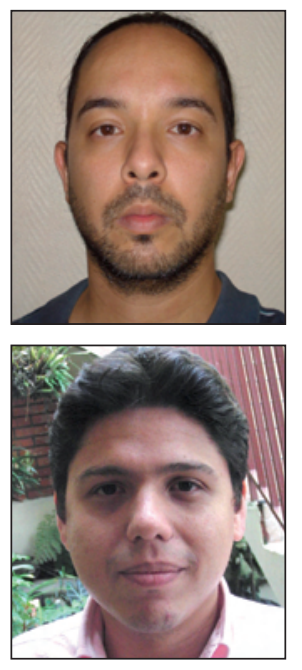

Elías Said-Hung es sociólogo por la Universidad Central de Venezuela y doctor por la Universidad Complutense de Madrid en tecnología, estructura y tratamiento de la información. Actualmente se desempeña como docente/investigador del Departamento de Comunicación Social y Periodismo, y director del Observatorio de Educación del Caribe Colombiano de la Universidad del Norte en Barranquilla, Colombia.

Universidad del Norte, Barranquilla, Colombia saide@uninorte.edu.co

Carlos Arcila-Calderón, comunicólogo, especialista en comunicación digital, es profesor del Departamento de Comunicación Social y Periodismo de la Universidad del Norte en Barranquilla e investigador asociado del Centro de Investigaciones de la Comunicación (CIC) de la Universidad Católica Andrés Bello (UCAB). Director del Anuario Electrónico de Estudios en Comunicación Social "Disertaciones". Doctor europeo en comunicación, cambio social y desarrollo por la Universidad Complutense de Madrid.

Universidad del Norte, Barranquilla, Colombia carcila@gmail.com

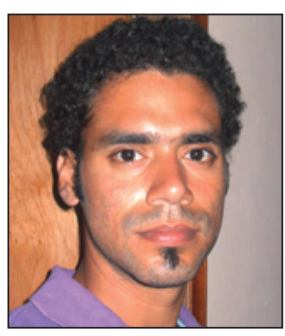

Jorge Méndez-Barraza es comunicador social egresado de la facultad de Comunicación Social y Periodismo de la Universidad del Norte en Barranquilla. Realizador audiovisual de Caja de Cartón Comunicación Visual y asistente de investigaciones del Observatorio de Educación del Caribe Colombiano de la Universidad del Norte en Barranquilla, Colombia.

Universidad del Norte, Barranquilla, Colombia jorgemendez.023@gmail.com

\section{Resumen}

Se analizan 38 diarios digitales de Colombia con el fin de determinar la evolución de los cibermedios en dicho país, prestando especial atención a la incorporación de las herramientas de la web 2.0. El trabajo parte del proyecto "Los cibermedios en Colombia y América Latina", de la Universidad del Norte en Colombia, con el apoyo de la Universidad de los Andes en Venezuela en 2010, en el que se aplica el modelo de análisis propuesto por Rodríguez-Martínez, Codina y Pedraza-Jiménez (2010) para elaborar un indicador para medir el desarrollo de los diarios digitales en Colombia. Los resultados apuntan que tienen bajos niveles de accesibilidad, visibilidad y popularidad, así como baja utilización de herramientas de interacción, profundización y personalización de los contenidos. Se concluye con un ranking de calidad.

\section{Palabras clave}

Diarios digitales, Prensa digital, Ciberperiodismo, Medios de comunicación, Web 2.0, Colombia.

\section{Title: The development of Colombian online newspapers}

\begin{abstract}
In this study we analyse 38 online newspapers in Colombia, with the goal of determining the level of development of cybermedia in this country, taking special account of the incorporation of Web 2.0 tools. This work is part of a bigger project, "Cybermedia in Colombia and Latin America", which is being coordinated by the Universidad del Norte (Colombia), with the recent support of the Universidad de los Andes (Venezuela). For the empirical research, we applied the model proposed by Rodríguez-Martínez, Codina y Pedraza-Jiménez (2010), with the further objective of building an indicator to measure the development of Colombian online newspapers. The results show that these digital media have low levels of accessibility, visibility and popularity, and therefore low use of tools for interaction, in-depth exploration and personalization of contents. The study concludes with a quality ranking of cybermedia in Colombia.
\end{abstract}




\section{Keywords}

Online newspapers, Online media, Cyber journalism, Mass communication, Web 2.0, Colombia.

Said-Hung, Elías; Arcila-Calderón, Carlos; Méndez-Barraza, Jorge. "Desarrollo de los cibermedios en Colombia". El profesional de la información, 2011, enero-febrero, v. 20, n. 1, pp. 47-53...

DOI: 10.3145/epi.2011.ene.06

\section{Introducción}

La acelerada trasformación de los medios de comunicación en internet y el creciente nivel de exigencia de los usuarios hace necesaria una evaluación constante de los cibermedios, especialmente si tomamos en cuenta la incorporación de herramientas que permiten una participación más activa de los usuarios. En América Latina, como en otras partes del planeta, la evolución de los cibermedios ha estado marcada por una limitada utilización de los recursos propios del medio digital; sin embargo parece haber un compromiso por superar esta situación, derivado de la necesidad de los medios por adaptarse a los nuevos mercados de consumidores de información. En países como Colombia los cibermedios están experimentando constantes cambios en sus formatos y contenidos con el fin de proveer a sus visitantes de servicios informativos de calidad.

La medición y el análisis de los cibermedios es un tema recurrente en la literatura científica, tanto en Estados Unidos (Schultz, 1999) como en Europa (Van-der-Wurff; Lauf; O'Sullivan, 2005). En América Latina destacan algunos trabajos de países como Brasil (Palacios et al., 2002), México (López-Aguirre, 2009) y Venezuela (Orejuela, 2009), pero aun cuando existen esfuerzos en marcha (Castro, 2008) no hay trabajos completos que realicen un seguimiento exhaustivo de la evolución de los cibermedios en la región. Esta realidad se debe en parte al escaso desarrollo teórico y metodológico sobre el tema. No obstante, en este campo cabe señalar algunas propuestas concretas como la cibergrafía de Cely (2004), los avances metodológicos de DíazNoci (1997), Palacios y Diaz-Noci (2009) y Zamith (2008), o el método de análisis de Rodríguez-Martínez, Codina y Pedraza-Jiménez (2010), del cual parte este trabajo.

\section{Los cibermedios están experimentando constantes cambios para proveer a sus visitantes de servicios informativos de calidad}

\section{Objetivos y Metodología}

Con este trabajo se pretende analizar el grado de adopción del ciberperiodismo en Colombia, mediante la elaboración de un indicador que contribuya a la medición de la calidad de los medios digitales de este país. Este análisis se aplicó en 38 diarios digitales activos en mayo de 2010, según el listado de prensa escrita en Colombia. Con los diarios analizados se abarcan todos los ámbitos de difusión en Colombia: nacional, regional, departamental y local (tabla 1). Los medios analizados fueron:
- nacionales: El tiempo, El espectador, El espacio, La república, Portafolio, El colombiano;

- regionales: El heraldo, La patria, La nación, El diario de Huila, El diario del sur, La crónica del Quindío, El país, El diario de occidente, La tarde, La vanguardia;

- departamentales: El nuevo siglo, El nacional, El periódico, El mundo, La opinión, El universal, El liberal, El pilón, El meridiano de Córdoba, El diario del norte, El diario del Magdalena, El informador, El diario, El frente, El meridiano de Sucre;

- locales: Diario deportes, La libertad, El diario de Magangue, El comunicador, Q'Hubo, Extra, ADN.

http://www.prensaescrita.com/america/colombia.php

Se tomaron como diarios de control las versiones online de The New York times de Estados Unidos y El país de España, que se encuentran entre los principales medios de comunicación digitales según el ranking de Alexa: The New York times se encontraba el 25 de abril de 2010 en el puesto 93 de las webs con mayor tráfico a nivel mundial, y 23 de Estados Unidos; y El país estaba en el puesto 548 mundial y 16 de España.

\begin{tabular}{|l|c|c|}
\hline & $\begin{array}{c}\text { Número de diarios } \\
\text { digitales analizados }\end{array}$ & $\%$ \\
\hline Local & 7 & 18,4 \\
\hline Departamental & 15 & 39,5 \\
\hline Regional & 10 & 26,3 \\
\hline Nacional & 6 & 15,8 \\
\hline Total & 38 & 100,0 \\
\hline
\end{tabular}

Tabla 1. Ámbitos de difusión

Para el estudio se utilizó el modelo de análisis propuesto por Rodríguez-Martínez, Codina y Pedraza-Jiménez (2010) y se tomaron como referentes un conjunto de indicadores:

1) Generales, que miden la accesibilidad del sitio web, su visibilidad y popularidad, entre los aspectos que toda web de un diario digital debe cuidar.

2) Específicos internos, que dan cuenta de la profundización de la información, las herramientas de interacción empleadas en los diarios digitales, así como la personalización de la información, y de aquellos elementos que el sitio de un diario digital debiese incluir.

3) Específicos externos, relacionados con el impacto de las webs sociales en los diarios digitales, en el que se miden los mecanismos de fidelización de los usuarios mediante las plataformas de la web 2.0 y otras herramientas dispuestas en los diarios, así como los mecanismo de difusión de los contenidos generados en los mismos. 
El estudio, que forma parte del proyecto Los cibermedios en Colombia y América Latina (adelantado desde la Universidad del Norte en Colombia, con el apoyo de la Universidad de los Andes en Venezuela en 2010), analizó la página principal y todas las secciones de los 38 diarios digitales seleccionados en Colombia. La medición se realizó en días alternos (de lunes a domingo), a lo largo de un mes de análisis, desde el 25 de abril hasta el 25 de mayo de 2010. Durante este período se realizaron mediciones de repetición y verificación, que garantizaron la consistencia de los datos.

\section{Resultados}

\subsection{Indicadores generales de los cibermedios en Co- lombia}

\section{Accesibilidad}

Según los autores citados (Rodríguez-Martínez et al., 2010), "idealmente, para llegar a la mayor cantidad posible de lectores e informarles, un diario debería implementar su web conforme a un nivel de adecuación AAA". A partir de la medición de los errores detectados automáticamente por el Test de accesibilidad web (Taw), en cada sitio y para cada nivel de adecuación, los resultados muestran tres grandes grupos: sitios web sin errores o con un número reducido (menor a 45); sitios con un número de errores moderado o similar al de los diarios de control (45-85 errores); sitios con un gran número (de 85 a 200) o con un excesivo número de errores (más de 200). El promedio del total sitúa a los cibermedios colombianos con un excesivo número de errores $(252,2)$.

\section{http://www.tawdis.net}

Si bien observamos que la accesibilidad de estos sitios no es buena, algunos de los cibermedios estudiados alcanzaron los requisitos del nivel de Prioridad I, especialmente los departamentales y regionales. Los medios con un nivel de accesibilidad bueno son: El nacional (6 errores), Diario del Magdalena (7) y La opinión (9), entre los departamentales; y Diario del Huila (23), La patria (30) y Diario del sur (31), entre los regionales. Estos datos los ubican muy por encima de los diarios de control El país (45) y The New York times (85), que tienen un número de errores moderado.

\section{Visibilidad y popularidad}

Pueden ser medidas por el número de enlaces que recibe el medio de otros sitios web, por el número de páginas publicadas e indexadas, así como por el número de visitas que recibe el sitio y el número de páginas servidas por éste.

Como vemos en la tabla 2, los datos obtenidos de los 38 diarios digitales de Colombia, nos permiten ubicarlos con un bajo nivel de visibilidad y popularidad. Salvando los enlaces recibidos en Yahoo! Search a nivel departamental donde se aprecia un número superior a lo obtenido en el caso de los diarios digitales regionales, la visibilidad y popularidad de los contenidos de los diarios variarán proporcionalmente de acuerdo con el ámbito de difusión que posee cada uno de los medios analizados.

\section{Acceso a la información}

Uno de los indicadores generales es la capacidad del medio de brindar un acceso rápido y fácil a la información. Se to-

\begin{tabular}{|c|c|c|c|c|}
\hline & $\begin{array}{l}N^{\circ} \text { de } \\
\text { diarios }\end{array}$ & Mínimo & Máximo & Media \\
\hline \multicolumn{5}{|c|}{ Datos del conjunto' ${ }^{1}$} \\
\hline $\begin{array}{l}\text { PageRank de } \\
\text { Google* }\end{array}$ & 39 & 0 & 10 & 4,6 \\
\hline Ranking mundial & 38 & 0 & 2.408 .889 & 377.288 \\
\hline Ranking nacional & 38 & 0 & 14.687 & 2.588 \\
\hline $\begin{array}{l}\text { Enlaces recibidos } \\
\text { Yahoo! Search }\end{array}$ & $37^{2}$ & 0 & 6.370 .000 & 557.749 \\
\hline $\begin{array}{l}\text { Páginas indexadas } \\
\text { Yahoo! Search }\end{array}$ & 38 & 1 & 808.000 & 56.574 \\
\hline \multicolumn{5}{|c|}{ Diarios digitales de control Nytimes.com y Elpais.com } \\
\hline $\begin{array}{l}\text { PageRank de } \\
\text { Google* }\end{array}$ & 2 & 8 & 9 & 8,5 \\
\hline Ranking mundial & 2 & 92 & 548 & 320 \\
\hline Ranking nacional & 2 & 16 & 23 & 19 \\
\hline $\begin{array}{l}\text { Enlaces recibidos } \\
\text { Yahoo! Search }\end{array}$ & 2 & 15.400 .000 & 126.000 .000 & 70.700 .000 \\
\hline $\begin{array}{l}\text { Páginas indexadas } \\
\text { Yahoo! Search }\end{array}$ & 2 & 5.950 .000 & 15.600 .000 & 10.775 .000 \\
\hline \multicolumn{5}{|l|}{ Nacional } \\
\hline $\begin{array}{l}\text { PageRank de } \\
\text { Google* }\end{array}$ & 6 & 0 & 10 & 6,6 \\
\hline Ranking mundial & 6 & 1.995 & 371.556 & 82.806 \\
\hline Ranking nacional & 6 & 11 & 1.755 & 379 \\
\hline $\begin{array}{l}\text { Enlaces recibidos } \\
\text { Yahoo! Search }\end{array}$ & 6 & 19 & 3.280 .000 & 1.219 .036 \\
\hline $\begin{array}{l}\text { Páginas indexadas } \\
\text { Yahoo! Search }\end{array}$ & 6 & 3 & 808.000 & 240.705 \\
\hline \multicolumn{5}{|l|}{ Regional } \\
\hline $\begin{array}{l}\text { PageRank de } \\
\text { Google* }\end{array}$ & 10 & 0 & 10 & 5,8 \\
\hline Ranking mundial & 10 & 19.980 & 896.379 & 195.261 \\
\hline Ranking nacional & 10 & 84 & 14.687 & 2.569 \\
\hline $\begin{array}{l}\text { Enlaces recibidos } \\
\text { Yahoo! Search }\end{array}$ & $9^{2}$ & 1 & 3.740 .000 & 437.233 \\
\hline $\begin{array}{l}\text { Páginas indexadas } \\
\text { Yahoo! Search }\end{array}$ & 10 & 3 & 198.000 & 51.724 \\
\hline \multicolumn{5}{|l|}{ Departamental } \\
\hline $\begin{array}{l}\text { PageRank de } \\
\text { Google* }\end{array}$ & 15 & 0 & 10 & 4,8 \\
\hline Ranking mundial** & 15 & 0 & 1.041 .997 & 343.394 \\
\hline Ranking nacional ${ }^{* *}$ & 15 & 0 & 6.792 & 2.115 \\
\hline $\begin{array}{l}\text { Enlaces recibidos } \\
\text { Yahoo! Search }\end{array}$ & 15 & 1 & 6.370 .000 & 583.014 \\
\hline $\begin{array}{l}\text { Páginas indexadas } \\
\text { Yahoo! Search }\end{array}$ & 15 & 3 & 87.500 & 11.931 \\
\hline \multicolumn{5}{|l|}{ Local } \\
\hline $\begin{array}{l}\text { PageRank de } \\
\text { Google* }\end{array}$ & 7 & 0 & 10 & 2,9 \\
\hline Ranking mundial & 7 & 0 & 2.408 .889 & 962.373 \\
\hline Ranking nacional & 7 & 0 & 13.374 & 5.518 \\
\hline $\begin{array}{l}\text { Enlaces recibidos } \\
\text { Yahoo! Search }\end{array}$ & 7 & 0 & 611.000 & 91.740 \\
\hline $\begin{array}{l}\text { Páginas indexadas } \\
\text { Yahoo! Search }\end{array}$ & 7 & 1 & 6.180 & 1.337 \\
\hline
\end{tabular}

* Min $=0$ (ninguna visibilidad) / Máx = 10 (visibilidad óptima)

* Se excluyen en este análisis los diarios de los que no había datos disponibles en el momento de realizar el estudio.

Notas:

1. No se incluyen en este cálculo del total los diarios digitales tomados como control.

2. Se excluye para el cálculo a El país de Colombia por disposición investigativa ya que su alto número de enlaces recibidos de Yahoo! Search, a diferencia del número observado en el resto de medios digitales regionales generan distorsión en la media de este apartado.

Tabla 2. Visibilidad y popularidad a nivel general y según difusión 


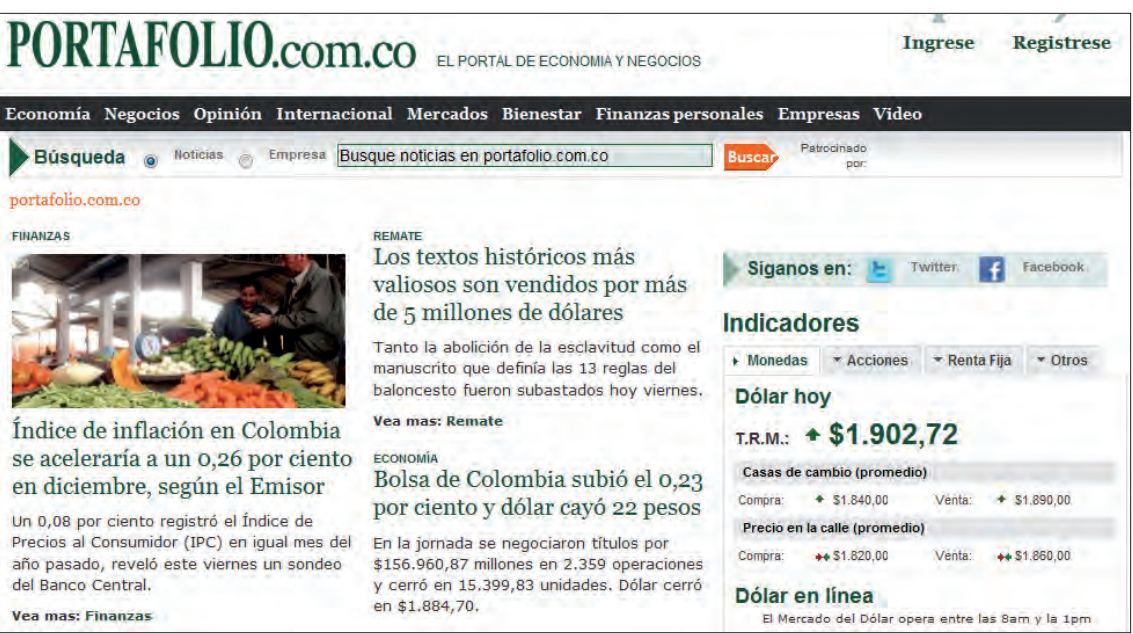

Portafolio, http://www.portafolio.com.co/

maron como referentes cuatro aspectos: búsqueda simple, búsqueda avanzada, hemeroteca y mapa del sitio web. Los resultados no son nada positivos (tabla 3): está generalizada la búsqueda simple (65,7\% de los 38 diarios analizados), pero la mayoría no tiene alguno de los restantes elementos de análisis (búsqueda avanzada, hemeroteca y mapa del sitio web). Los medios que ofrecen mayor posibilidad de acceso a la información a sus usuarios son El tiempo, El universal, y La vanguardia.

\begin{tabular}{|c|r|r|r|r|r|r|r|r|}
\hline & \multicolumn{2}{|c|}{$\begin{array}{c}\text { Búsqueda } \\
\text { simple }\end{array}$} & \multicolumn{2}{|c|}{$\begin{array}{c}\text { Búsqueda } \\
\text { avanzada }\end{array}$} & \multicolumn{2}{|c|}{ Hemeroteca } & \multicolumn{2}{|c|}{$\begin{array}{c}\text { Mapa del } \\
\text { sitio web }\end{array}$} \\
\cline { 2 - 10 } & $\begin{array}{c}\text { No de } \\
\text { dia- } \\
\text { rios }\end{array}$ & $\%$ & $\begin{array}{c}|c| \\
\text { No de } \\
\text { dia- } \\
\text { rios }\end{array}$ & $\%$ & $\begin{array}{c}\text { No de } \\
\text { dia- } \\
\text { rios }\end{array}$ & $\%$ & $\begin{array}{c}\text { No de } \\
\text { dia- } \\
\text { rios }\end{array}$ & $\%$ \\
\hline Sí & 25 & 65,7 & 7 & 18,4 & 4 & 10,5 & 8 & 21,1 \\
\hline No & 13 & 34,2 & 31 & 81,5 & 34 & 89,5 & 30 & 78,9 \\
\hline N & 38 & 100,0 & 38 & 100,0 & 38 & 100,0 & 38 & 100,0 \\
\hline$\mu$ & \multicolumn{2}{|c|}{1,37} & \multicolumn{2}{|c|}{1,84} & \multicolumn{2}{|c|}{1,89} & \multicolumn{2}{|c|}{1,79} \\
\hline
\end{tabular}

Tabla 3. Diarios con búsqueda simple, búsqueda avanzada, hemeroteca y mapa del sitio web

\section{El $90 \%$ de los medios que no usan la web} 2.0 ejercen su labor periodística a nivel local y/o departamental en Colombia

\subsection{Indicadores específicos internos}

Según Rodríguez-Martínez et al., para el análisis del grado de adopción del ciberperiodismo y de la web 2.0 en la prensa digital hay que estudiar las particularidades de cada uno de los medios analizados. Estos autores proponen veinticinco indicadores, que en este estudio fueron ampliados a treinta y siete, manteniéndose su agrupación en tres categorías: profundización de la información, herramientas de interacción, y personalización de la información.

\section{Profundización de la información}

Uno de los rasgos que caracterizan a los medios digitales es la capacidad de especialización de los contenidos elaborados y divulgados por ellos (García, 2004). Los resultados obtenidos (tabla 4) muestran que los diarios digitales en
Colombia presentan un bajo nivel de profundización de la información, al comparar las medias del número de enlaces totales a artículos de la misma sección de la noticia aparecida en la portada, número de noticias totales con enlace a hemeroteca, y número de noticias que emplean audio o vídeo, respecto a los diarios de control. Los niveles de los diarios colombianos solamente son similares a los diarios de control en enlaces externos y en uso de fotografía/infografía.

\section{Herramientas de interacción}

En este apartado se ha pasado de los 9 indicadores originales propuestos por Rodríguez-Martínez et al., a 18. Entre los indicadores originales están: seguimiento estadístico de noticias, empleo y creación de blogs, inclusión de comentarios, disposición de herramientas web 2.0, y existencia de foros; y se han añadido nuevos como: existencia de la sección noticia más valorada y noticia más enviada, oportunidades de votación, y nivel de participación de los usuarios.

Los resultados (tabla 5) permiten señalar el bajo nivel de empleo de herramientas para la participación de los usuarios en los diarios digitales en Colombia. Se puede destacar por tanto la persistencia de perspectivas periodísticas tradicionales en la prensa digital colombiana, en las que el usuario mantiene los rasgos de pasividad propios de la prensa impresa.

\section{Personalización de la información}

Como exponen Hume (1996), Briggs (2007), Franco (2007), Flores (2008), o Méndez-Rubio (2004), los avances de las TICs han contribuido a cambiar el discurso monológico des-

\begin{tabular}{|l|c|c|c|c|}
\hline & $\begin{array}{c}\text { No de } \\
\text { diarios }\end{array}$ & $\begin{array}{c}\text { Míni- } \\
\text { mo }\end{array}$ & $\begin{array}{c}\text { Máxi- } \\
\text { mo }\end{array}$ & Media \\
\hline $\begin{array}{l}\text { Enlaces totales a artículos } \\
\text { de la sección por noticias } \\
\text { aparecidas en portada }\end{array}$ & 38 & 0 & 40 & 5,32 \\
\hline $\begin{array}{l}\text { Noticias totales con enla- } \\
\text { ces a hemeroteca digital }\end{array}$ & 38 & 0 & 0 & 0 \\
\hline $\begin{array}{l}\text { Noticias con enlaces } \\
\text { externos }\end{array}$ & 38 & 0 & 35 & 3,0 \\
\hline $\begin{array}{l}\text { Noticias totales mostradas } \\
\text { en portada con Fotografía/ } \\
\text { Infografía }\end{array}$ & 38 & 0 & 24 & 8,6 \\
\hline $\begin{array}{l}\text { Noticias que emplean } \\
\text { audio o vídeo }\end{array}$ & 38 & 0 & 15 & 1,6 \\
\hline \multicolumn{2}{|c|}{ Diarios digitales de control Nytimes.com y Elpais.com } \\
\hline $\begin{array}{l}\text { Enlaces totales a artículos } \\
\text { de la sección por noticias } \\
\text { aparecidas en portada }\end{array}$ & 2 & 14 & 20 & 17,0 \\
\hline $\begin{array}{l}\text { Noticias totales con enla- } \\
\text { ces a hemeroteca digital }\end{array}$ & 2 & 0 & 17 & 8,5 \\
\hline $\begin{array}{l}\text { Noticias con enlaces } \\
\text { externos }\end{array}$ & 2 & 0 & 4 & 2,0 \\
\hline $\begin{array}{l}\text { Noticias totales mostradas } \\
\text { en portada con Fotografía/ } \\
\text { Infografía }\end{array}$ & 2 & 4 & 14 & 9,0 \\
\hline $\begin{array}{l}\text { Noticias que emplean } \\
\text { audio o vídeo }\end{array}$ & 2 & 3 & 6 & 4,50 \\
\hline
\end{tabular}

Tabla 4. Profundización de la información 


\begin{tabular}{|c|c|c|c|c|}
\hline & sí & $\%$ & NO & $\%$ \\
\hline $\begin{array}{l}\text { ¿Dispone de herramientas de segui- } \\
\text { miento estadístico de la noticia? }\end{array}$ & 2 & 5,3 & 36 & 94,7 \\
\hline ¿Dispone de un blog? & 12 & 31,6 & 26 & 68,4 \\
\hline $\begin{array}{l}\text { ¿Ofrece a los usuarios la posibilidad } \\
\text { de crear un blog dentro de su propio } \\
\text { dominio? }\end{array}$ & 2 & 5,3 & 36 & 94,7 \\
\hline $\begin{array}{l}\text { ¿Se pueden hacer comentarios a las } \\
\text { noticias publicadas? }\end{array}$ & 24 & 63,2 & 14 & 36,8 \\
\hline $\begin{array}{l}\text { ¿Dispone de herramientas web } 2.0 \\
\text { para compartir contenidos? }\end{array}$ & 18 & 47,4 & 20 & 52,6 \\
\hline $\begin{array}{l}\text { ¿Permite a los lectores contactar vía } \\
\text { correo electrónico con el autor de una } \\
\text { noticia? }\end{array}$ & 9 & 23,7 & 29 & 76,3 \\
\hline $\begin{array}{l}\text { ¿Acepta contribuciones de los } \\
\text { lectores? }\end{array}$ & 9 & 23,7 & 29 & 76,3 \\
\hline ¿Dispone de foros de discusión? & 5 & 13,2 & 33 & 86,8 \\
\hline $\begin{array}{l}\text { ¿Dispone de opción para el envío de } \\
\text { correcciones de las noticias? }\end{array}$ & 2 & 5,3 & 36 & 94,7 \\
\hline ¿Dispone de comentarios las noticias? & 20 & 52,6 & 18 & 47,4 \\
\hline ¿Tiene chats para sus usuarios? & 2 & 5,3 & 36 & 94,7 \\
\hline $\begin{array}{l}\text { ¿Posee sección con las noticias más } \\
\text { leídas? }\end{array}$ & 10 & 26,3 & 28 & 73,7 \\
\hline $\begin{array}{l}\text { ¿Posee sección con las noticias más } \\
\text { valoradas? }\end{array}$ & 5 & 13,2 & 33 & 86,8 \\
\hline $\begin{array}{l}\text { ¿Posee sección con las noticias más } \\
\text { enviadas? }\end{array}$ & 1 & 2,6 & 37 & 97,4 \\
\hline $\begin{array}{l}\text { ¿Tiene opción de votación de la } \\
\text { noticia? }\end{array}$ & 8 & 21,0 & 30 & 79,0 \\
\hline
\end{tabular}

Tabla 5. Herramientas de interacción

de los medios de comunicación digitales y han propiciado el surgimiento de una nueva audiencia más participativa, no anónima e independiente, habilitada para procesos de intercambio y feedback más consolidados entre periodistausuario. Todo ello aparece enmarcado en un contexto dominado por internet, en el que convergen diferentes tipos de comunicación: pasiva, unívoca y centrada en el emisor (Llorca, 2005); así como el acceso a un volumen mayor de información, de modo personalizado, en función de intereses de grupos de usuarios muy pequeños y hasta individuales, los cuales difícilmente podrían ser atendidos bajo el modelo de los medios de comunicación tradicionales (Díaz-Noci, 1997).

Sin embargo, como se aprecia en la tabla 6, en los diarios colombianos las oportunidades de seguimiento y aceptación de contenidos por parte de los usuarios, así como los aspectos vinculados con la instauración de nuevas rutinas periodísticas están aún muy lejos de generalizarse.

\subsection{Indicadores externos}

Con la llegada de la web 2.0, según autores como Franco (2007), Fumero y Roca (2007), Rodríguez-Martínez, Codina y Pedraza-Jiménez (2010), y Codina (2010), entre otros muchos investigadores, se abre un nuevo escenario en el que se cuenta con la oportunidad de ampliación de la comunidad de usuarios alrededor de los diarios digi-

\begin{tabular}{|l|r|r|r|c|}
\hline & \multicolumn{1}{|c|}{ Sí } & \multicolumn{1}{c|}{$\%$} & \multicolumn{1}{c|}{ NO } & $\%$ \\
\hline ¿Posee servicio de alerta? & 7 & 18,4 & 31 & 81,6 \\
\hline ¿Dispone de RSS? & 19 & 50,0 & 19 & 50,0 \\
\hline $\begin{array}{l}\text { ¿Es posible el envío de noticias vía } \\
\text { correo electrónico? }\end{array}$ & 20 & 52,6 & 18 & 47,4 \\
\hline ¿Es posible la impresión de la noticia? & 20 & 52,6 & 18 & 47,4 \\
\hline $\begin{array}{l}\text { ¿Facilita el envío de noticias a teléfo- } \\
\text { nos móviles? }\end{array}$ & 2 & 5,3 & 36 & 94,7 \\
\hline ¿Ofrece la opción de registrarse? & 18 & 47,4 & 20 & 52,6 \\
\hline $\begin{array}{l}\text { ¿Cuenta con aplicaciones que per- } \\
\text { mitan a sus usuarios personalizar los } \\
\text { contenidos del diario? }\end{array}$ & 2 & 5,3 & 36 & 94,7 \\
\hline
\end{tabular}

Tabla 6. Personalización de la información

tales y las noticias producidas por los periodistas, a favor del aumento de mecanismos de atracción, fidelización y difusión de contenidos más allá de los portales web de los medios. A diferencia de lo que plantean Rodríguez-Martínez et al., para la medición de la inclusión de las herramientas de la web 2.0 se propone aquí un análisis que permite no sólo medir el empleo de dichas herramientas en los diarios digitales colombianos, sino también la presencia de otros portales o plataformas dentro del cibermedio.

\section{Los diarios digitales en Colombia presen- tan un bajo nivel de profundización de la información}

Los resultados de este trabajo nos muestran un bajo uso de las herramientas de la web 2.0 . El $50 \%$ de los periódicos analizados no emplean este tipo de recursos para la divulgación de sus contenidos, bien sea permitiendo a sus usuarios agregar o compartir contenidos, o divulgar desde estos portales sus contenidos como usuarios registrados. Es importante destacar además que el $90 \%$ de los medios que no usan herramientas de la web 2.0 son mayoritariamente diarios que ejercen su labor periodística a nivel local y/o departamental, lo cual puede estar incidiendo negativa-

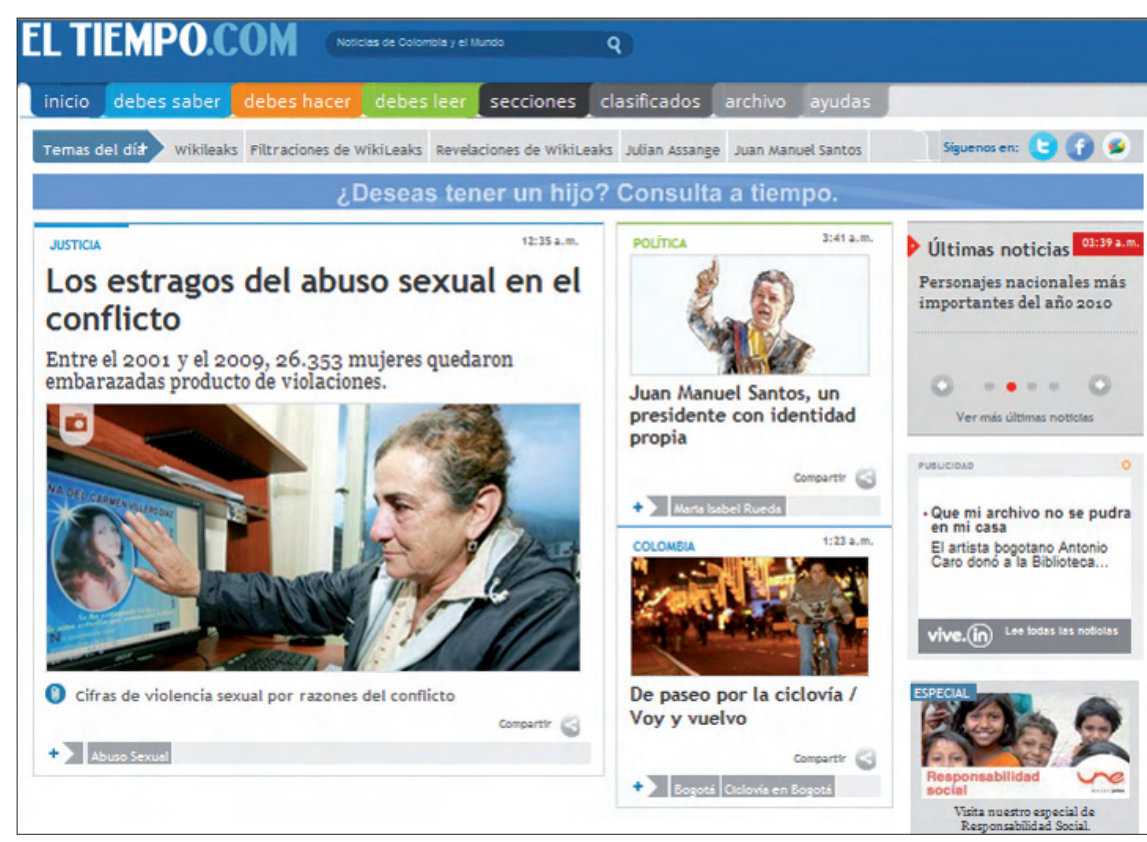

El tiempo.com, http://www.eltiempo.com/ 
mente en sus capacidades actuales de aumentar su impacto en sus esferas de acción social, así como en la toma de contacto con un mayor número de usuarios potencialmente interesados en acceder a las noticias divulgadas desde internet.

\section{Propuesta de ranking de cibermedios en Colombia}

A partir de los resultados obtenidos se ha intentado establecer un ranking que permita vislumbrar el grado de evolución de los cibermedios en este país. Para esta medición llevamos a cabo el siguiente cálculo: DC-Col (Desarrollo de cibermedios en Colombia) = IG (Indicadores generales) + IEi (Indicadores específicos internos) + IExt (Indicadores externos - web 2.0). La totalidad de los apartados que forman parte del DC-Col darán consigo un valor $X$ sobre 100 puntos, correspondientes al total de puntos pautados para la medición de lo aquí expuesto (tabla 7).

El peso de cada apartado varió en relación con el número de variables pautadas en cada uno, distribuyéndose los 100 puntos como se muestra a continuación:

- Indicadores generales (IG): 27 puntos máximos, de los cuales: 3 puntos correspondieron a los errores de accesibilidad; 20 puntos fueron asignados a todo lo referente a la visibilidad y popularidad de los diarios; y 4 se asignaron a la presencia o no de buscadores simples, buscadores avanzados, hemeroteca y mapa del sitio web.

- Indicadores específicos internos (IEi): 50 puntos distribuidos en: 28 para las variables de profundización de la información; 15 para las variables de uso de herramientas de interacción; y 7 para las variables de medición de la capacidad de personalización de la información.

- Indicadores externos (IExt): 24 puntos totales distribuidos en: 24 para aquellos diarios que hacen uso de herramientas propias de la web 2.0 para permitir a sus usuarios compartir información y divulgar ésta en otras plataformas.

\section{Conclusiones}

Los medios de la tabla 7 se ubican en puestos privilegiados de liderazgo en Colombia, quedan-
Tabla 7. Ranking de los Top 10 cibermedios en Colombia

\begin{tabular}{|l|c|c|c|c|c|}
\hline \multicolumn{1}{|c|}{ Cibermedio } & $\begin{array}{c}\text { IG } \\
\text { (máximo } \\
\text { 27 puntos) }\end{array}$ & $\begin{array}{c}\text { IÉ } \\
\text { (máximo } \\
50 \text { puntos) }\end{array}$ & $\begin{array}{c}\text { IExt } \\
\text { (máximo } \\
23 \text { puntos) }\end{array}$ & $\begin{array}{c}\text { DC-Col } \\
\text { (máximo } \\
100 \text { puntos) }\end{array}$ & Posición \\
\hline $\begin{array}{l}\text { Portafolio } \\
\text { http://www.portafolio.com.co/ }\end{array}$ & 23,0 & 28,5 & 23 & 74,5 & 1 \\
\hline $\begin{array}{l}\text { El tiempo } \\
\text { http://www.eltiempo.com/ }\end{array}$ & 18,5 & 29,5 & 23 & 71,0 & 2 \\
\hline $\begin{array}{l}\text { El colombiano } \\
\text { http://www.elcolombiano.com/ }\end{array}$ & 17,0 & 25,5 & 23 & 65,5 & 3 \\
\hline $\begin{array}{l}\text { El universal } \\
\text { http://www.eluniversal.com.co/ }\end{array}$ & 18,0 & 19,5 & 23 & 60,5 & 4 \\
\hline $\begin{array}{l}\text { La vanguardia } \\
\text { http://www.vanguardia.com/ }\end{array}$ & 19,0 & 18,5 & 23 & 60,5 & 4 \\
\hline $\begin{array}{l}\text { La república } \\
\text { http://www.larepublica.com.co/ }\end{array}$ & 15,0 & 15,0 & 23 & 53,0 & 5 \\
\hline $\begin{array}{l}\text { El espacio } \\
\text { http://www.elespacio.com.co/ }\end{array}$ & 15,5 & 14,0 & 23 & 52,5 & 6 \\
\hline $\begin{array}{l}\text { El heraldo } \\
\text { http://www.elheraldo.com.co/ }\end{array}$ & 16,5 & 13,0 & 23 & 52,5 & 6 \\
\hline $\begin{array}{l}\text { Nuevo siglo } \\
\text { http://www.elnuevosiglo.com.co/ }\end{array}$ & 14,0 & 11,5 & 23 & 48,5 & 7 \\
\hline $\begin{array}{l}\text { El país } \\
\text { http://www.elpais.com.co/ }\end{array}$ & 13,5 & 11,0 & 23 & 47,5 & 8 \\
\hline $\begin{array}{l}\text { La patria } \\
\text { http://www.lapatria.com/ }\end{array}$ & 7,5 & 15,0 & 23 & 45,5 & 9 \\
\hline $\begin{array}{l}\text { La nación } \\
\text { http://www.lanacion.com.co/ }\end{array}$ & 11,5 & 11,0 & 23 & 45,5 & 9 \\
\hline $\begin{array}{l}\text { El espectador } \\
\text { http://www.elespectador.com/ }\end{array}$ & 9,0 & 19,5 & 12 & 40,5 & 10 \\
\hline
\end{tabular}

do claro que aún queda mucho camino por avanzar para poder aumentar los valores obtenidos. Debemos tener en cuenta que las cabeceras que lideran el escenario de cibermedios en este país aún siguen perdiendo importantes puntos tanto en los indicadores específicos internos (en especial en la profundización de la información y las herramientas de interacción) como en indicadores externos (es decir, en el nivel y forma de empleo de las plataformas de la web 2.0 en dichos medios).

Los resultados señalan que si bien el desarrollo de los cibermedios colombianos aún es insuficiente, algunos tienen 
una mejor situación en algunos indicadores que nuestros medios control (El país y The New York times), por ejemplo en lo relativo a la accesibilidad, lo que sugiere que las dinámicas en estos medios están forzando las transformaciones en materia de calidad.

El ranking propuesto en este artículo así como el detalle de los indicadores, contribuye a la descripción del estado de los cibermedios en Colombia y sienta un punto de referencia para la futura comparación con el resto de países de América Latina.

Los primeros diarios en el ranking son Portafolio (74,5/100 puntos), El tiempo (71), El colombiano $(65,5)$, El universal $(60,5)$, La vanguardia $(60,5)$

\section{Bibliografía}

Briggs, Mark. Periodismo 2.0. Una guía de alfabetización digital, 2007.

http://knightcenter.utexas.edu/Periodismo_20.pdf

Castro, Cosette. Industrias de contenidos en Latinoamérica. Documento de Grupo de Trabajo eLAC2007. Enero, 2008.

Cely, Adriana. “Cibergrafía: propuesta teórico metodológica para el estudio de los medios de comunicación social cibernéticos". Opción, 2004, año 20, n. 43, pp. 101-118.

http://redalyc.uaemex. $m x / s r c /$ inicio/ArtPdfRed.jsp?iCve= 31004306

Codina, Lluís. "Ciencia 2.0: redes sociales y aplicaciones en línea para académicos". Hipertext.net, 2009, n. 7.

http://www.upf.edu/hipertextnet/numero-7/ciencia-2-0. $--h t m l$

Díaz-Noci, Javier. "Tendencias del periodismo electrónico. Una aproximación a la investigación sobre medios de comunicación en internet". Zer, 1997, n. 2.

http://www.ehu.es/zer/zer2/6artdiaz.html

Flores, Jesús. "El blog band de la información". En: Flores, Jesús; Cebrián, Mariano; Esteve Francisco (Eds). Blogalaxia y periodismo en la Red. Estudios, análisis y reflexiones. Madrid: Editorial Fragua, 2008.

Franco, Guillermo. Cómo escribir para la Web. Bases para la construcción y discusión de manuales de redacción online, 2007.

http://knightcenter.utexas.edu/como_web.php

Fumero, Antonio; Roca, Genís. Web 2.0. Madrid, España: Fundación Orange, 2007.

García, Elvira. "La especialización en la era de internet". En: Fernández-Del-Moral, Javier (Coord.). Periodismo especializado, Madrid: Ariel, 2004, pp. 195-218.
Hume, Ellen. "The new paradigm for news". Annals of the American Academy of Political and Social Science, 1996, v. 546, n. 1 , pp. 141-153.

Llorca, Germán. “Comunicación interpersonal y comunicación de masas en internet. Emisor y receptor en el entorno virtual". En: López-García, Guillermo (coord.). El ecosistema digital. Modelos de comunicación, nuevos medios y público en internet. Valencia: Universidad de Valencia, 2005. pp. 2129.

López-Aguirre, José-Luis. "Estudio de servicios y productos documentales en la prensa digital mexicana". Documentación de las ciencias de la información, 2009, v. 32, pp. 93132.

http://revistas.ucm.es/inf/02104210/articulos/DCIN090911 0093A.PDF

Méndez-Rubio, Antonio. Perspectivas sobre comunicación y sociedad. Valencia: Universidad de Valencia, 2004.

Orejuela, Merlyn. "Del usuario selector al usuario comunicador: oferta, uso y aprovechamiento de los recursos interactivos en El-nacional.com y El-universal.com". En: IV Congreso de cibersociedad, 2009.

Palacios, Marcos; Mielniczuk, Luciana; Barbosa, Suzana; Ribas, Beatriz; Narita, Sandra. "Um mapeamento de características e tendências no jornalismo online brasileiro e português". Comunicarte, 2002, septiembre, v. 1, n. 2.

Palacios, Marcos; Díaz-Noci, Javier (Eds.). Online journalism: research methods. A multidisciplinary approach in comparative perspective. Bilbao: Servicio Editorial de la Universidad del País Vasco, 2009.

http://www.argitalpenak.ehu.es/p291-content/es/conte nidos/libro/se_indice_ciencinfo/es_ciencinf/adjuntos/ journalism.pdf

Quesada, Montse. “Periodismo especializado”. En: Galdón, Gabriel (Coord.). Introducción a la comunicación y a la información. Barcelona: Ariel, 2001.

Rodríguez-Martínez, Ruth; Codina, Lluís; Pedraza-Jiménez, Rafael. "Cibermedios y web 2.0: modelo de análisis y resultados de aplicación". El profesional de la información, 2010, enero-febrero, v. 19, n. 1, pp. 35-44.

Schultz, Tanjev. "Interactive options in online journalism: a content analysis of 100 U.S. newspapers". Journal of computer-mediated communication, 1999, Sept., v. 5, n. 1. http://jcmc.indiana.edu/vol5/issue1/schultz.html

Van-der-Wurff, Richard; Lauf, Edmund; O'Sullivan, John (eds.). Print and online newspapers in Europe: a comparative content analysis in 16 countries in Western and Eastern Europe. Amsterdam: Het Spinhuis, 2005.

Zamith, Fernando. "A methodological proposal to analyze the news websites use of the potentialities of the internet". En: 9th Intl symposium on online journalism, 2008. 